\title{
AMBIENTE DE TERAPIA INTENSIVA PEDIÁTRICA: \\ IMPLICAÇÕES PARA A ASSISTÊNCIA DA CRIANÇA E DE SUA FAMÍLIA
}

\author{
ENVIRONMENT OF PEDIATRIC INTENSIVE CARE: \\ IMPLICATIONS FOR THE ASSISTANCE OF THE \\ CHILD AND THEIR FAMILY
}

\section{ENTORNO DE CUIDADOS INTENSIVOS PEDIÁTRICOS: IMPLICACIONES PARA LA ASISTENCIA DEL NIÑO Y SU FAMILIA}

\author{
Soraya Bactuli Cardoso ${ }^{1}$ \\ Isabel Cristina dos Santos Oliveira ${ }^{2}$ \\ Elena Araújo Martinez ${ }^{3}$ \\ Sandra Alves do Carmo ${ }^{4}$ \\ Rita de Cássia Melão de Moraes ${ }^{5}$ \\ Mauro César de Oliveira Santos
}

Como citar este artigo: Cardoso SB, Oliveira ICS, Martinez EA, Carmo SA, Moraes RCM, Santos MCO. Ambiente de terapia intensiva pediátrica: implicações para a assistência da criança e de sua família. Rev baiana enferm. 2019;33:e33545.

Objetivo: caracterizar a produção científica nacional e internacional acerca do ambiente da Unidade de Terapia Intensiva Pediátrica e sua influência na assistência à criança e à sua família. Método: revisão integrativa realizada de setembro a dezembro de 2018. As fontes de informação foram: LILACS, SciELO, CINAHL, PubMed e CAPES, com recorte temporal emergindo da busca (2004 a 2018). Resultados: os 38 estudos selecionados apresentaram o ambiente em sua estrutura física; ambiente como influenciador nas condutas da equipe de saúde e direcionamento da assistência; ambiente como espaço de trocas de experiências e vivências; e ambiente como mediador de relações entre equipe de enfermagem, criança e sua família. Conclusão: o ambiente da Unidade de Terapia Intensiva Pediátrica deve contemplar conforto e privacidade para a criança e sua família, além de proporcionar autonomia para a equipe.

Descritores: Unidade de Terapia Intensiva Pediátrica. Arquitetura Hospitalar. Criança Hospitalizada. Enfermagem Pediátrica. Ambiente de Instituições de Saúde.

\footnotetext{
Enfermeira. Mestre em Enfermagem. Enfermeira do Instituto Nacional de Saúde da Mulher, da Criança e do Adolescente Fernandes Figueira. Universidade Federal do Rio de Janeiro. Rio de Janeiro, RJ, Brasil. bactuli@yahoo.com.br. https://orcid.org/0000-0002-4665-242

2 Enfermeira. Mestre em Enfermagem Pediátrica e Pediatria Social. Doutora em Enfermagem. Professora da Universidade Federal do Rio de Janeiro. Rio de Janeiro, RJ, Brasil. https://orcid.org/0000-0003-2916-1396

3 Enfermeira. Mestre em Enfermagem. Doutora em Enfermagem. Enfermeira do Instituto Nacional de Saúde da Mulher, da Criança e do Adolescente Fernandes Figueira. Rio de Janeiro, RJ, Brasil. https://orcid.org/0000-0002-3I55-I02X

4 Enfermeira. Mestre em Enfermagem. Enfermeira do Instituto Nacional de Câncer. Universidade Federal do Rio de Janeiro. Rio de Janeiro, RJ, Brasil. https://orcid. org/0000-000 I-84/3-0053

Enfermeira. Doutora em Enfermagem. Professora da Universidade de Brasilia. Brasília, Distrito Federal, Brasil. https://orcid.org/0000-000 I-8526-0642

6 Arquiteto. Doutor em Arquitetura. Professor da Universidade Federal do Rio de Janeiro. Rio de Janeiro, RJ, Brasil. http://orcid.org/0000-0002-4093-0830
} 
Objective: to characterize the national and international scientific production on the environment of the Pediatric Intensive Care Unit and its influence on the assistance to the child and their family. Method: integrative review conducted from September to December 2018. The sources of information were: LILACS, SciELO, CINAHL, PubMed and CAPES, with temporal clipping emerging from the search (2004 to 2018). Results: the 38 selected studies showed the environment in its physical structure; environment as influencer of the health team's behavior and targeting of assistance; environment as a space of exchanges of experiences; and the environment as a mediator of relations between nursing staff, child and their family. Conclusion: the environment of a Pediatric Intensive Care Unit should contemplate privacy and comfort for the child and their family, in addition to providing autonomy to the team.

Descriptors: Intensive Care Units, Pediatric. Hospital Design and Construction. Child, Hospitalized. Pediatric Nursing. Health Facility Environment.

Objetivo: caracterizar la producción científica nacional e internacional sobre el ambiente de la Unidad de Cuidados Intensivos Pediátricos y su influencia en la atención a niños y sus familias. Método: revisión integradora, llevada a cabo de septiembre a diciembre de 2018. Fuentes de información: LILACS, SciELO, CINAHL, PUbMed y CAPES, con recorte temporario de la búsqueda (2004 a 2018). Resultados: 38 estudios seleccionados presentaron el ambiente en su estructura física; ambiente como factor de influencia en la conducta del equipo de salud y dirección de la atención; como espacio de intercambio de experiencias; y como mediador de las relaciones entre personal de enfermería, niños y familias. Conclusión: el ambiente de la Unidad de Cuidados Intensivos Pediátricos debe incluir comodidad y privacidad para niño y familia, asi como proporcionar autonomía para el equipo.

Descriptores: Unidades de Cuidado Intensivo Pediátrico. Arquitectura y Construcción de Hospitales. Niño Hospitalizado. Enfermería Pediátrica. Ambiente de Instituciones de Salud.

\section{Introdução}

A Unidade de Terapia Intensiva (UTI) é idealizada com base nas ações de Florence Nightingale. Em 1854 ocorreu a guerra da Crimeia, na qual Inglaterra, França e Turquia declararam guerra à Rússia. Os soldados vinham a óbito pelas condições precárias, porém a taxa de mortalidade reduziu com intervenções de cuidados mais complexos e especializados, isto é, foram classificados de acordo com o grau de gravidade. Desse modo, os mais graves ficavam próximos à enfermagem, com monitorização contínua. Sendo assim, o objetivo básico da unidade de terapia intensiva é recuperar ou dar suporte às funções vitais dos pacientes em um ambiente físico e psicológico adequado ${ }^{(1)}$.

A primeira Unidade de Terapia Intensiva Pediátrica e Neonatal (UTIPN) do Brasil, foi implementada somente em 1974 no Sabará Hospital Infantil em São Paulo, com o que havia de mais moderno em equipamentos de monitoramento cardiopulmonar e ventilação mecânica, apoiados numa equipe médica associada aos avanços diagnósticos e terapêuticos da época ${ }^{(2)}$.

Ao longo dos anos, avanços tecnológicos vêm modificando o ambiente da Unidade de Terapia
Intensiva Pediátrica (UTIP), o que é uma tendência mundial. Contudo, as crianças convivem com o estresse e a dor devido às terapias invasivas decorrentes da tecnologia. Esta, cada vez mais avançada, gera desconforto físico e mental, além de causar impacto negativo no cuidado(3).

A UTI é um local com equipamentos de tecnologia de ponta, destinado a pacientes que necessitam de cuidados complexos e monitorização contínua. Caracterizada por ser um ambiente inóspito, com ruídos, alarmes, iluminação constante, realização de procedimentos invasivos e movimentação de profissionais, torna-se ainda mais depressor e estressor ao paciente ${ }^{(1)}$. Esse ambiente depressor e de estresse intensifica-se durante a hospitalização de uma criança, uma vez que repercute no emocional de todos os que estão envolvidos: criança, família e equipe de saúde ${ }^{(4)}$.

A preocupação com a influência do ambiente hospitalar no processo de recuperação do paciente iniciou-se com Florence Nightingale, no século XIX, e está registrada em seu livro "Notas sobre Enfermagem - o que é e o que não é". Escrito em 1859 e traduzido para o português em 
1989, conceitua ambiente hospitalar como local no qual se encontram o doente e/ou familiares, e compreende as instituições de saúde e o domicílio, além de considerar a interrelação entre os componentes físico, social e psicológico ${ }^{(5)}$.

Dessa forma, Florence Nightingale, em sua teoria ambientalista, considerava importantes alguns elementos para a manutenção de um ambiente saudável, tais como: ventilação, provisão de ar fresco e puro; iluminação, claridade e luz solar direta; calor, relacionado a evitar o resfriamento dos pacientes; limpeza, referente à prevenção de infecções; ruídos, necessidade de observância do silêncio; odores e alimentação ${ }^{(5)}$. Além disso, destacava que variedades de objetos, formas e cores também contribuíam positivamente para a boa recuperação mental e física durante a internação ${ }^{(5)}$.

Entretanto, até os dias atuais, esses elementos ambientais ainda não são plenamente considerados pelos profissionais da área de saúde. Destaca-se a área de terapia intensiva, em especial a pediátrica, visto que ainda se observam unidades extremamente frias, janelas fechadas e a utilização de persianas que não possibilitam a entrada de iluminação natural nem a percepção do dia/noite.

Nesses ambientes, as cores são monótonas, muitas vezes sem nenhuma ou com poucas caracterizações infantis, e o nível de ruído é elevado. Há casos, em que o espaço físico dessas unidades não possibilita a presença do acompanhante, por não propiciar condições adequadas para seu repouso, ainda que o art. 12 da Lei n. 8.069, de 13 de julho de 1990, reelaborada pela Lei n. 13.257 , de 8 de março de 2016, que institui o Estatuto da Criança e do Adolescente (ECA), disponha que os estabelecimentos de atendimento à saúde, inclusive as unidades neonatais, de terapia intensiva e de cuidados intermediários, deverão proporcionar condições para a permanência em tempo integral de um dos pais ou responsável, nos casos de internação de criança ou adolescente $^{(6)}$.

No que se refere às políticas públicas vigentes relacionadas com a temática do ambiente, em 2003, foi criada a Política Nacional de Humanização (PNH), que compreende a ambiência na saúde como o espaço físico, social, profissional e de relações interpessoais que deve estar relacionado a um projeto de saúde voltado para a atenção acolhedora, resolutiva e humana. A ideia de ambiência segue primordialmente três eixos: a ambiência como espaço de encontros entre os sujeitos, a produção de saúde e de subjetividades; e o espaço como ferramenta facilitadora do processo de trabalho; e o espaço que visa à confortabilidade ${ }^{(7)}$.

A humanização de um edifício hospitalar é resultante de um processo projetual amplo, que não se limita à beleza, mas diz respeito à funcionalidade, ao domínio dos aspectos construtivos que favoreça a recuperação da saúde e garanta o bem-estar físico e psicológico aos usuários, sejam eles pacientes, acompanhantes ou funcionários $^{(8)}$. Para isso, esse projeto precisa apresentar uma perfeita combinação entre os conceitos da arquitetura, da tecnologia, do conforto e bem-estar, em consonância com as leis e orientações dos órgãos de saúde ${ }^{(8)}$. A contribuição da arquitetura para a saúde infantil é fundamental, pois a concepção de projetos arquitetônicos de ambientes de saúde para a criança deve prever que o espaço seja projetado para ela. Desse modo, a hospitalização pode ser entendida de forma mais positiva ${ }^{(9)}$.

A realização deste estudo justifica-se pela importância de traçar um panorama em relação à ambiência da UTIP, que dará alicerce aos conhecimentos da equipe de enfermagem acerca da influência do ambiente para a assistência à criança e à sua família nesse ambiente, promovendo, assim, um cuidado mais humanizado e acolhedor. Destaca-se que a ambiência da UTIP deve oferecer conforto, bem-estar e segurança às crianças e às suas famílias, além de propiciar autonomia da equipe, possibilitando a utilização do próprio espaço como ferramenta facilitadora de produção de saúde, o que confere a importância da investigação sobre a temática e sua relevância para o âmbito da ciência e da sociedade.

O objetivo desta pesquisa é caracterizar a produção científica nacional e internacional acerca 
do ambiente da Unidade de Terapia Intensiva Pediátrica (UTIP) e sua influência na assistência à criança e à sua família.

\section{Método}

Optou-se pelo método de revisão integrativa, que consiste na construção de uma análise ampla da literatura, obtendo-se um conhecimento profundo de um determinado fenômeno baseado em estudos anteriores ${ }^{(10)}$.

Este tipo de revisão é elaborado em seis etapas: identificação do tema e seleção da questão de pesquisa, estabelecimento dos critérios de inclusão e exclusão para a seleção das amostras, categorização dos estudos, avaliação dos estudos incluídos na revisão, interpretação dos resultados e apresentação da revisão ${ }^{(10)}$.

Sendo assim, a revisão partiu da seguinte questão norteadora: Qual a influência do ambiente da unidade de terapia intensiva pediátrica na assistência à criança e à sua família?

A busca, realizada entre setembro e dezembro de 2018, iniciou-se com a seleção de descritores (DeCS) pertinentes às temáticas: humanização da assistência, unidades de terapia intensiva pediátrica, criança hospitalizada, enfermagem pediátrica, arquitetura hospitalar, ambiente de instituições de saúde, planejamento hospitalar, reestruturação hospitalar e meio ambiente e saúde ambiental. Estes descritores foram conjugados em pares, utilizando-se o operador boleano and, para efeito de busca, nas seguintes fontes de informações: Literatura Latino Americana e do Caribe em Ciências da Saúde (LILACS), Scientific Eletronic Library Online (SciELO), Coordenação de Aperfeiçoamento de Pessoal de Nivel Superior (CAPES), Cumulative Index to Nursingand Allied Health Literature (CINAHL) e Public Medline (PubMed).

Para a seleção dos estudos, foram determinados os seguintes critérios de inclusão: artigos de pesquisa, pesquisas de revisão, pesquisas histórica e documental, dissertações e teses, nos idiomas português, inglês e espanhol, disponíveis na íntegra e desenvolvidos em unidades de terapia intensiva pediátrica.
Os critérios de exclusão foram relatos de experiência, estudos clínicos, pesquisas realizadas em unidades de tratamento intensivo pediátricas especializadas em áreas como oncologia, cardiologia e ortopedia, uma vez que, nessas unidades, o tratamento dado ao ambiente já é diferenciado, devido à cronicidade das doenças e ao tempo de internação das crianças.

O recorte temporal foi aberto, resultando no ano de 2004, quando se identificou a primeira publicação acerca da temática nas fontes de informações, até o ano de 2018.

Vale ressaltar que, no portal de teses e dissertações da CAPES, quando utilizada a mesma combinação de descritores, e conforme realizado nas outras fontes de informação, foi obtido como resultado mais de 20.000 estudos. Sendo assim, optou-se por utilizar somente os seguintes descritores: arquitetura hospitalar, ambiente de instituições de saúde, planejamento hospitalar e reestruturação hospitalar, sem conjugação, todos em caixa alta e entre aspas.

Após a busca e a aplicação dos critérios de inclusão e exclusão, foi realizada a leitura dos resumos. Para facilitar o processo de análise temática, os estudos incluídos foram organizados em um quadro sinóptico com a finalidade de obter-se uma síntese das informações, tais como título, referência, objetivos, categoria dos autores, tipo de estudo, participantes/amostra, cenário e procedência. Entretanto, para a apresentação neste artigo, foi preciso reduzi-lo.

Após a organização dos artigos selecionados inscritos no quadro sinóptico, foram feitas leituras flutuantes, recortes de interesse, atendendo ao objeto de investigação, identificadas categorias e subcategorias temáticas e estabelecida a classificação por nível de evidência.

O estudo em questão não foi submetido à apreciação de Comitê de Ética em Pesquisa, pois não utilizou seres humanos como sujeitos de pesquisa.

\section{Resultados}

O levantamento bibliográfico inicial resultou em um total de 2.137 estudos. Dois revisores 
independentes realizaram a leitura de títulos, resumos e descritores, com vistas a verificar se as publicações apresentavam adequação ao objetivo do estudo. Foram aplicados os critérios de inclusão e exclusão, sendo selecionadas 97 pesquisas, que apresentaram aderência à temática do ambiente da UTIP e a influência na assistência à criança e à sua família. Posteriormente, foi realizada a eliminação de 59 publicações repetidas nas fontes de informações, resultando em 38 publicações selecionadas, conforme Figura 1.

Figura 1 - Diagrama ilustrado do caminhar metodológico para a identificação dos estudos selecionados

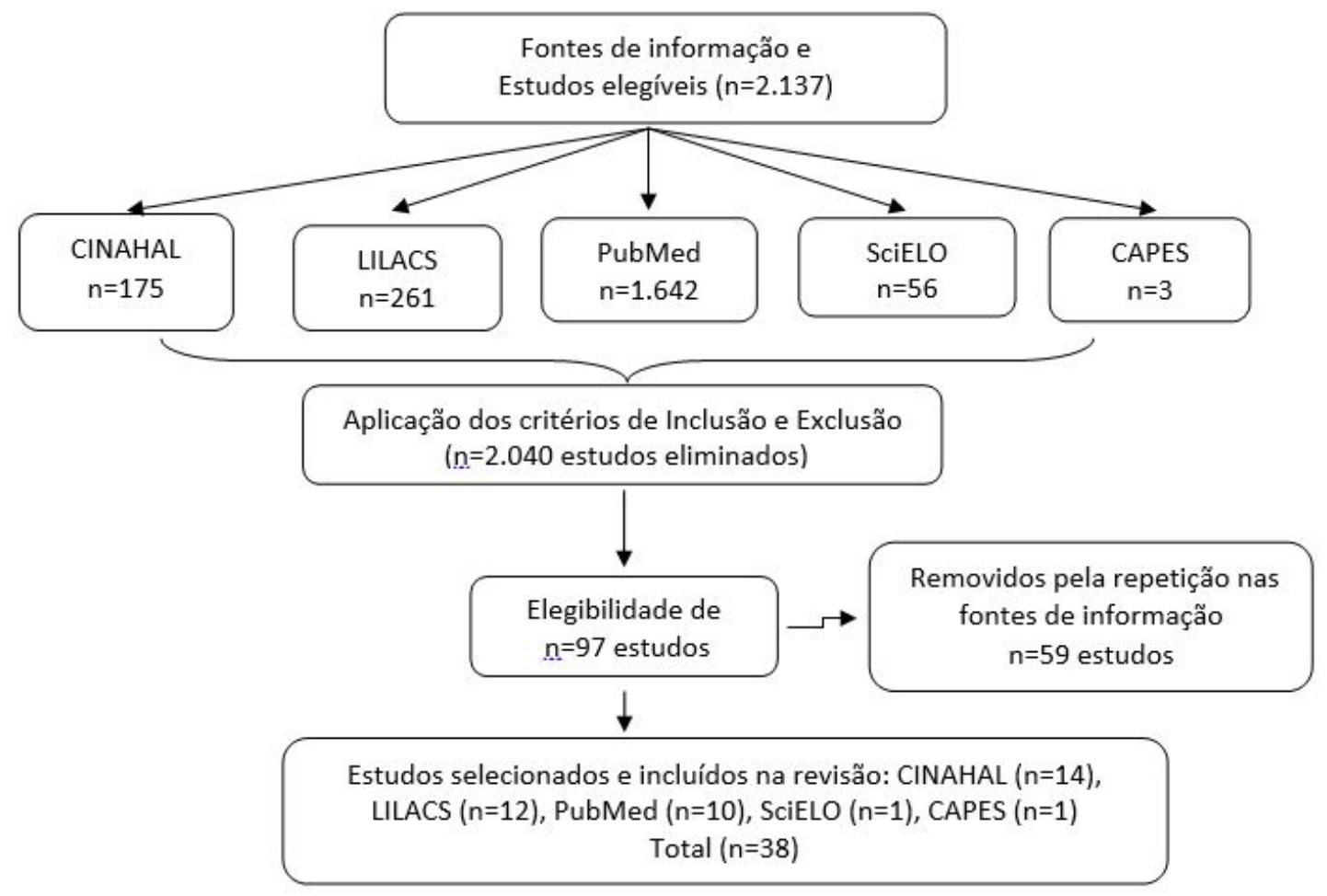

Fonte: Elaboração própria.

O Quadro 1 apresenta os 38 artigos selecionados segundo título, periódico e ano de publicação do mais recente ao mais antigo.

Quadro 1 - Seleção de Publicações sobre o ambiente da Unidade de TerapiaIntensiva Pediátrica (continua)

\begin{tabular}{|l|l|c|}
\hline Título & \multicolumn{1}{|c|}{ Periódico } & Ano \\
\hline $\begin{array}{l}\text { Pediatric Critical care in Resource-Limited Settings - } \\
\text { Overview and lessons learned }\end{array}$ & Frontiers in Pediatrics & 2018 \\
\hline $\begin{array}{l}\text { Estresores Parentales em Unidad de Cuidados Intensivos } \\
\text { Pediátricos }\end{array}$ & Rev Chil Pediatr & 2018 \\
\hline $\begin{array}{l}\text { Nurses' reflections on benefits and challenges of } \\
\text { implemmenting family-centered care in pediatric intensive } \\
\text { care units }\end{array}$ & Am J Crit Care & 2018 \\
\hline $\begin{array}{l}\text { Metassíntese sobre o homem como pai e cuidador de um } \\
\text { filho hospitalizado }\end{array}$ & Rev Latino-Am Enfermagem & 2017 \\
\hline Missed Nursing care in Pediatrics & Hospital Pediatrics & 2017 \\
\hline $\begin{array}{l}\text { Humanização da assistência de enfermagem à família na } \\
\text { UTIP }\end{array}$ & Cogitare Enferm & 2016 \\
\hline
\end{tabular}


Quadro 1 - Seleção de Publicações sobre o ambiente da Unidade de TerapiaIntensiva Pediátrica (continua)

\begin{tabular}{|c|c|c|}
\hline Título & Periódico & Ano \\
\hline $\begin{array}{l}\text { UTI pediátrica: o significado do cuidar na perspectiva da } \\
\text { mãe }\end{array}$ & $\begin{array}{l}\text { Rev Fund Care Online de } \\
\text { Pesquisa }\end{array}$ & 2016 \\
\hline $\begin{array}{l}\text { UTIP: vidas pulsando entre a estabilidade orgânica e a } \\
\text { instabilidade subjetiva }\end{array}$ & Tese de doutorado. UFMG & 2016 \\
\hline $\begin{array}{l}\text { Parents' perceived satisfaction of care, communication } \\
\text { and enviroment of the Pediatric Intensive Care Units at a } \\
\text { Tertiary Children's Hospital }\end{array}$ & Journal of Pediatric Nursing & 2016 \\
\hline $\begin{array}{l}\text { Characterising the ambient sound enviroment for infants } \\
\text { in intensive care wards }\end{array}$ & $\begin{array}{l}\text { Journal of Paediatrics and Child } \\
\text { Health }\end{array}$ & 2016 \\
\hline $\begin{array}{l}\text { Nurses' perceptions of pediatric intensive care unit } \\
\text { enviroment and work experience after transition to single- } \\
\text { patient rooms }\end{array}$ & $\begin{array}{l}\text { American Journal of Critical } \\
\text { Care }\end{array}$ & 2016 \\
\hline $\begin{array}{l}\text { Ambiente de trabalho da enfermagem, segurança do } \\
\text { paciente e qualidade do cuidado em hospital pediátrico }\end{array}$ & Rev Gaúcha Enferm & 2016 \\
\hline $\begin{array}{l}\text { Comparison of staff and family perceptions of causes of } \\
\text { noise pollution in the pediatric intensive care unit and } \\
\text { suggested intervention strategies }\end{array}$ & Noise Health & 2016 \\
\hline $\begin{array}{l}\text { Dominance of paternalism in family-centered care in } \\
\text { pediatric Intensive care unit: an ethnografhic study }\end{array}$ & $\begin{array}{l}\text { Comprehensive Pediatric } \\
\text { Nursing }\end{array}$ & 2015 \\
\hline Evidence-Based Review and Discussion points & $\begin{array}{l}\text { American Journal of Critical } \\
\text { Care }\end{array}$ & 2015 \\
\hline Impact of noise on nurses in pediatric intensive care units & $\begin{array}{l}\text { American Journal of Critical } \\
\text { Care }\end{array}$ & 2015 \\
\hline $\begin{array}{l}\text { What impact do hospital and unit-based rules have upon } \\
\text { patient and family-centered care in the pediatric intensive } \\
\text { care unit? }\end{array}$ & J Pediatr Nurs & 2015 \\
\hline $\begin{array}{l}\text { Ambiente de trabalho da enfermagem e segurança do } \\
\text { paciente em unidades pediátricas }\end{array}$ & Tese de doutorado UNICAMP & 2015 \\
\hline $\begin{array}{l}\text { Ambiência como estratégia de humanização da assistência } \\
\text { na unidade de pediatria: revisão sistemática }\end{array}$ & Rev Esc Enferm USP & 2014 \\
\hline $\begin{array}{l}\text { A presença do pai/acompanhante no âmbito hospitalar: } \\
\text { revisão integrativa }\end{array}$ & Rev Enferm UFPE on line & 2014 \\
\hline $\begin{array}{l}\text { The lived experience of parents of children admittes to } \\
\text { the pediatric intensive care unit in Lebanon }\end{array}$ & $\begin{array}{l}\text { International Journal of Nursing } \\
\text { studies }\end{array}$ & 2014 \\
\hline $\begin{array}{l}\text { Percepção da equipe de enfermagem sobre humanização } \\
\text { em UTIPN }\end{array}$ & Rev Gaúcha Enfermagem & 2013 \\
\hline Nível de ruído da UTIP: estudo observacional & $\begin{array}{l}\text { Online Brazilian Journal of } \\
\text { Nursing }\end{array}$ & 2013 \\
\hline $\begin{array}{l}\text { The experience of critically ill children: a } \\
\text { phenomenological study of discomfort and comfort }\end{array}$ & Dynamics & 2013 \\
\hline $\begin{array}{l}\text { An Office or a bedroom? Challenges for family-centered } \\
\text { care in the pediatric intensive care unit }\end{array}$ & Journal of child health care & 2012 \\
\hline $\begin{array}{l}\text { Factors influencing sleep for parents of critically ill } \\
\text { hospitalised children: A qualitative analysis }\end{array}$ & $\begin{array}{l}\text { Intensive and Critical Care } \\
\text { Nursing }\end{array}$ & 2011 \\
\hline $\begin{array}{l}\text { Refletindo sobre a inserção da família no cuidado à } \\
\text { criança hospitalizada }\end{array}$ & Rev Enferm UERJ & 2010 \\
\hline $\begin{array}{l}\text { Humanização: representações sociais do hospital } \\
\text { pediátrico }\end{array}$ & Rev Gaúcha Enfermagem & 2009 \\
\hline $\begin{array}{l}\text { Sofrimento psíquico interferindo no desejo materno de } \\
\text { cuidar do filho internado na UTIP }\end{array}$ & $\begin{array}{l}\text { Online Brazilian Journal of } \\
\text { Nursing }\end{array}$ & 2009 \\
\hline
\end{tabular}


Quadro 1 - Seleção de Publicações sobre o ambiente da Unidade de TerapiaIntensiva Pediátrica (conclusão)

\begin{tabular}{|l|l|c|}
\hline Título & \multicolumn{1}{|c|}{ Periódico } & Ano \\
\hline $\begin{array}{l}\text { Estresse ocupacional e suas repercussões na qualidade de } \\
\text { vida de médicos e enfermeiros intensivistas pediátricos e } \\
\text { neonatais }\end{array}$ & Rev Bras Ter Intensiva & 2009 \\
\hline Psicologia da saúde e criança hospitalizada & $\begin{array}{l}\text { PSIC-Revista de Psicologia da } \\
\text { Vetor Editora }\end{array}$ & 2008 \\
\hline Parent bed spaces in the PICU: effect on parental stress & Pediatric Nursing & 2007 \\
\hline $\begin{array}{l}\text { Pratice guidelines for music interventions with } \\
\text { hospitalized pediatric patients }\end{array}$ & Journal of Pediatric Nursing & 2007 \\
\hline $\begin{array}{l}\text { Humanização hospitalar: satisfação dos profissionais de } \\
\text { um hospital pediátrico }\end{array}$ & Rev Bras Enferm & 2006 \\
\hline $\begin{array}{l}\text { Nível de ruídos em uma unidade de cuidados intensivos } \\
\text { pediátricos }\end{array}$ & J Pediatr (Rio J) & 2005 \\
\hline Noise levels in PICU: an evaluative study & Pediatric Nursing & 2005 \\
\hline Preventing ICU sydrome in children & Pediatric Nursing & 2004 \\
\hline $\begin{array}{l}\text { Guidelines and levels of care for pediatric intensive care } \\
\text { units }\end{array}$ & Pediatrics & 2004 \\
\hline
\end{tabular}

Fonte: Elaboração própria.

Do total de 38 publicações, 21 (55\%) eram no cenário internacional, contemplando países como Estados Unidos, Chile, Jordânia, Canadá, Líbano, Austrália, Inglaterra e Irã, e 17 (45\%) nacionais, sendo 36 (95\%) artigos e 2 (5\%) teses. No que se refere ao tipo de estudo, 15 (39\%) eram qualitativos, 11 (29\%) quantitativos, 9 (24\%) revisões, 2 (5\%) observacionais e 1 (3\%) não indicava o tipo de estudo no texto.

Os estudos foram avaliados quanto ao nível de evidência segundo a Oxford Centre for Evidence-Based Medicine. De acordo com esse critério, 17 (45\%) artigos eram de nível 4, 15 (39\%) de nível 2C e 6 (16\%) de nível $5^{(11)}$.
No que concerne aos participantes, 11 (29\%) foram realizados com equipe de saúde (enfermeiros, técnicos, auxiliares, médicos, fisioterapeutas, psicólogos); 10 (26\%) foram feitos com os pais; 1 (3\%) com crianças; 2 (5\%) utilizaram mais de uma categoria de participantes no momento da coleta, como, por exemplo, pais e equipe, ou equipe e crianças; e 14 (37\%) utilizaram outras formas de coleta de dados, tais como observações e revisão de literatura.

Após a leitura dos artigos selecionados, os estudos foram agrupados de acordo com a temática abordada e divididos em categorias e subcategorias para facilitar a discussão dos resultados, conforme Quadro 2.

Quadro 2 - Categorias e subcategorias temáticas

(continua)

\begin{tabular}{|l|l|}
\hline Categorias temáticas & \multicolumn{1}{c|}{ Subcategorias temáticas } \\
\hline Ambiente & $\begin{array}{l}\text { Ambiente como estratégia de humanização } \\
\text { Espaço físico: quarto individual/quarto compartilhado } \\
\text { Ruídos } \\
\text { Influência do ambiente no trabalho e na qualidade de vida da } \\
\text { equipe } \\
\text { Estrutura e organização da unidade }\end{array}$ \\
\hline Humanização & $\begin{array}{l}\text { Efeitos da hospitalização na criança } \\
\text { Inserção, participação da família e sua relação com a equipe } \\
\text { Percepção/satisfação da equipe } \\
\text { Satisfação da família em relação ao cuidado prestado à criança } \\
\text { Atividades lúdicas }\end{array}$
\end{tabular}


Quadro 2 - Categorias e subcategorias temáticas (conclusão)

\begin{tabular}{|l|l|}
\hline Categorias temáticas & \multicolumn{1}{|c|}{ Subcategorias temáticas } \\
\hline $\begin{array}{l}\text { Experiências e vivências da } \\
\text { hospitalização }\end{array}$ & Visão dos pais \\
& $\begin{array}{l}\text { Visão das crianças } \\
\text { Visão da equipe }\end{array}$ \\
\hline Cuidado centrado na família & Visão dos pais \\
\hline
\end{tabular}

Fonte: Elaboração própria.

\section{Discussão}

Encontrou-se, na literatura, um quantitativo importante de publicações que abordavam o ambiente da UTIP em seus resultados. Na busca das características da UTIP, foram identificadas quatro categorias temáticas como pontos relevantes e com destaque nas pesquisas. O ambiente foi apresentado com as seguintes perspectivas: ambiente em sua estrutura física; ambiente como influenciador nas condutas da equipe de saúde e direcionamento da assistência; ambiente como espaço de troca de experiências e vivências; e ambiente como mediador de relações entre a equipe de enfermagem, a criança e sua família.

Vale destacar que, dentre os elementos do ambiente, tais como ruídos, iluminação, temperatura, odores e ventilação, apenas o elemento ruído teve evidência nos estudos. Esse destaque configura uma limitação desses estudos em relação à configuração do ambiente da UTIP, visto que os demais elementos também apresentam consequências na recuperação dos pacientes, conforme a Teoria Ambientalista de Florence Nightingale.

A ambiência tem sido entendida como um pilar para a humanização da unidade pediátrica, pois é confirmada a importância de um espaço voltado para as crianças como auxílio no processo de cura. Ademais, enfatiza-se o uso da arquitetura como forma de proporcionar bem- estar à criança e à família, além de facilitar o desenvolvimento do processo de trabalho dos profissionais de saúde ${ }^{(9,12)}$.

Outro aspecto identificado foi a preferência por quartos individuais em substituição às unidades compartilhadas, uma vez que os enfermeiros relataram melhores condições da exposição à luz solar, além da melhoria no ciclo de sono-vigília das crianças. Além disso, os enfermeiros revelaram ter sido menor $\mathrm{O}$ ruído nos quartos individuais. Entretanto, os ruídos (alarmes dos monitores e as conversas da equipe) influenciaram negativamente o sono das crianças em ambas as configurações ${ }^{(13)}$.

No que se refere aos ruídos na UTIP, os estudos são unânimes em afirmar que ultrapassam as recomendações de órgãos nacionais (35 dBA a $45 \mathrm{dBA})^{(14-15)}$ e internacionais (30 dBA a $\left.40 \mathrm{dBA}\right)^{(16)}$, e os níveis mais elevados foram no período diurno. Afirma-se também que os maiores ruídos nessas unidades provêm dos profissionais de saúde, seguido dos alarmes dos equipamentos utilizados ${ }^{(17-23)}$. Enfatiza-se ainda que a exposição a altos níveis de ruído deixa os enfermeiros de unidades de cuidados críticos suscetíveis a ter sua produtividade afetada, prejudicando sua capacidade efetiva de tomar decisões e regular as emoções, o que afeta a qualidade do cuidado ${ }^{(20)}$.

No que concerne à influência do ambiente no trabalho e na qualidade de vida da equipe, observou-se que os profissionais percebiam o ambiente favorável à prática assistencial e à segurança do paciente. Em contrapartida, o ambiente de trabalho também foi considerado favorável à prática profissional, mas variáveis como exaustão emocional e satisfação no trabalho foram preditoras no quesito segurança ${ }^{(24-25)}$.

Em outro estudo, mais da metade das enfermeiras pediátricas entrevistadas afirmaram que já deixaram de executar algum cuidado em seu turno de trabalho devido à escassez de materiais/ equipamentos e locais de trabalho com número elevado de pacientes em relação ao número de 
enfermeiras, influenciando a segurança e a qualidade do cuidado prestado à criança ${ }^{(26)}$.

A qualidade de vida dos profissionais de saúde que trabalham na terapia intensiva foi investigada em um estudo, que identificou, entre médicos e enfermeiros que trabalhavam nessas unidades, altos esforços, muitas demandas psicológicas e físicas, além da insegurança no trabalho, com repercussão na qualidade de vida desses profissionais ${ }^{(27)}$. Além disso, observou-se que a UTIP é um território normatizado, onde os protocolos clínicos medeiam as relações de poder entre os profissionais, e existe um intenso envolvimento afetivo entre as crianças e os profissionais. Estes lidam com seus limites pessoais, com a morte e com vivências instáveis do dia a dia do trabalho ${ }^{(28)}$.

No tocante à estrutura e organização da UTIP, estudos fornecem algumas diretrizes mínimas para a unidade, tais como: organização e estrutura administrativa, pessoal, instalações e serviços hospitalares, drogas, equipamentos, treinamento e educação continuada com o objetivo de ajudar os hospitais a garantir um cuidado mais adequado ao paciente. Acrescenta-se ainda que, para criação de uma UTI, principalmente em ambientes de baixa renda, além de todo planejamento e recursos específicos, o principal investimento deve ser feito de forma permanente na equipe de enfermagem e médica, para prover um ambiente propício para uma assistência de qualidade $^{(29-30)}$.

No quesito humanização, é necessário prestar uma assistência pensando nos danos que a hospitalização pode causar à criança internada. Um estudo destacou a necessidade de maior atenção da equipe de psicologia às crianças internadas na UTIP, pois permanecem muito restritas ao leito, exigindo que os enfermeiros estejam constantemente conscientes dos aspectos do cuidado imediato, além do atendimento às suas necessidades físicas e segurança psicológica. Esse estudo afirma ainda que, na UTIP, é necessária a existência de protocolos claros em relação à sedação e analgesia, assim como à introdução de uma ferramenta de avaliação de dor adequada para as crianças ventiladas e sedadas ${ }^{(31-32)}$.
No que se refere à inserção e participação da família na UTIP, verificou-se que as mudanças ocorridas na organização das unidades pediátricas, para garantir essa inserção, ainda não se encontram bem definidas, necessitando de melhor organização da prática assistencial. Em contrapartida, o cuidado compartilhado no ambiente da UTIP proporcionou às mães o reconhecimento de sua função materna, normalmente perdida em virtude da hospitalização. Nesse caso, as mães relataram que se sentiam úteis e valorizadas quando inseridas nos cuidados do filho e reconheciam o papel da equipe de enfermagem como incentivadora do envolvimento materno nos cuidados durante a internação ${ }^{(33-34)}$.

Essa necessidade de a equipe reconhecer a função materna durante a hospitalização também é apreciada em outro estudo, segundo o qual a hospitalização gerava na mãe um aumento do sofrimento psíquico, de tal forma que ela podia sentir-se incapaz e sem vontade de cuidar do filho. Por isso, seria necessário que os profissionais de saúde dessem apoio a elas e aprendessem a lidar com essa situação ${ }^{(35)}$.

Apenas um estudo destacou o pai na qualidade de cuidador da criança durante a hospitalização, demonstrando que, durante esse processo, ocorre uma mudança na relação familiar e laboral e a paternidade fica reprimida no ambiente hospitalar ${ }^{(36)}$.

A necessidade de adequação do ambiente e de estruturas físicas para permanência dos acompanhantes no âmbito hospitalar é também identificada nos textos analisados. Estudo observou redução do estresse dos pais com a nova configuração da UTIP, que incluía cama para a permanência dos acompanhantes. Entretanto, os pais relataram que o espaço, com essa nova configuração, ficou muito reduzido. Além disso, outro estudo enfatizou a importância de orientar os pais em relação aos procedimentos realizados na unidade e alertá-los de que não seriam obrigados a permanecer na unidade, mesmo tendo local adequado para repouso ${ }^{(37-38)}$.

No que tange a esse aspecto, observou-se que os pais apresentavam dificuldade para decidir se permaneciam ou não à beira do leito durante 
a noite. Essa decisão, muitas vezes, baseava-se na condição da criança, confiança na equipe, apoios sociais disponíveis, além de como a decisão seria percebida pela equipe. Curiosamente, os pais não apresentavam nenhuma sugestão de como o pessoal do hospital poderia ajudar na resolução desse desafio ${ }^{(39)}$.

A permanência dos familiares na UTIP foi considerada pelos pais como problemática, tendo em vista a necessidade de deixarem a unidade, para usar o banheiro e fazer as refeições - era proibido comer à beira do leito -, pois gerava frustração, por deixarem a criança sozinha durante esses períodos. Esse estado dos pais aumentava no período noturno, pois a redução de pessoal significava atrasos em responder à solicitação dos pais para entrarem novamente na unidade. Além disso, os pais encontraram muita resistência para sua participação plena e ativa no cuidado aos seus filhos. Essas barreiras evidenciaram a hierarquia entre o enfermeiro e os pais durante a hospitalização ${ }^{(40)}$.

No tocante à satisfação da equipe com a humanização do ambiente da UTIP, detectou-se que o ambiente de trabalho era considerado confortável pelos profissionais para exercerem suas funções. No entanto, alguns relataram necessidade de melhorias, principalmente no apoio dado a eles, sugerindo treinamentos, melhoria do ambiente hospitalar e da qualidade do atendimento. Por outro lado, a questão de estrutura física, a falta de apoio ao profissional e o estímulo à motivação foram considerados como ações não humanizadas na instituição, reforçando a falta e o envolvimento da gestão institucional na implantação da $\mathrm{PNH}^{(41-42)}$.

O cuidado humanizado, na visão da família, perpassava conceitos como o bom relacionamento, educação, respeito, atenção e acolhimento. Para os familiares, a equipe de enfermagem prestava assistência humanizada com relação aos aspectos técnicos e de organização do ambiente. Em contrapartida, atitudes como distração, conversas pessoais e falta de gentileza, eram caracterizadas por eles como não humanizadas ${ }^{(43)}$.
Estudo corrobora os achados supracitados, ao informar que mais da metade dos pais entrevistados não estava satisfeita com o nível de ruído da UTIP, com o tempo gasto pelas enfermeiras à beira do leito e com a maneira de a equipe de saúde prepará-los para a admissão da criança ${ }^{(44)}$.

Em relação às atividades lúdicas na UTIP, em ambientes críticos, foi encontrado apenas um estudo que relatava a musicoterapia como uma forma de reforçar o uso da música como abordagem complementar e alternativa para acalmar, relaxar, distrair, sendo até mesmo considerada um sedativo e um alívio para a dor. Esse estudo afirmava ainda que, até a data do estudo, não existiam dados convincentes que indicassem quaisquer efeitos deletérios sobre os resultados dos pacientes, mesmo sendo crianças gravemente doentes ${ }^{(45)}$.

Dois estudos abordaram experiências e vivências dos pais em relação à hospitalização dos filhos. Em um deles, os pais classificaram-na como uma viagem ao desconhecido e descreveram sua experiência na UTIP como estranha, nova e misteriosa. Além disso, disseram que o ambiente da UTIP era diferente de todo o hospital, e que a necessidade de conforto levava-os a procurar um ambiente mais saudável. Ademais, foram evidenciadas as três dimensões de estressores pelos pais: a dimensão clínica, considerada a mais estressante; a emocional; e a comunicação com a equipe. Esta última foi considerada a menos estressante ${ }^{(46-47)}$.

Apenas um estudo demonstrou a experiência da hospitalização na visão das crianças. Estas citaram como elementos de desconforto: medos, preocupações, mágoas, intervenções invasivas, falta de pessoas importantes, ruídos excessivos e tédio. Em contrapartida, relataram elementos de conforto: visita dos pais e de amigos, presença do bicho de pelúcia favorito, atividades de entretenimento e lazer e pensamentos de ir para casa, onde seriam capazes de realizar atividades como correr, dormir e acordar de forma espontânea ${ }^{(48)}$.

No que tange ao cuidado centrado na família, um estudo etnográfico examinou a dinâmica deste em uma UTIP no Irã. As observações participantes possibilitaram identificar que essa 
unidade tem um fundo paternalista e a atmosfera social está longe dos conceitos do cuidado centrado na família, uma vez que o ambiente não era apropriado para as crianças, os pais eram frequentemente separados de seus filhos durante a internação, havia uma participação limitada dos pais em relação aos cuidados de seus filhos e a comunicação não era interativa ${ }^{(49)}$.

Esse estudo ainda aborda que o pessoal devia estar convencido de que, além de obter conhecimento, experiência e desenvolver habilidades de aplicação de alta tecnologia no atendimento das necessidades físicas das crianças em estado crítico, também devia considerar as necessidades emocionais dos pacientes e de suas famílias e construir comunicações eficientes e recíprocas, adotando os regulamentos direcionados ao cuidado centrado na família, como, por exemplo, acompanhamento dos pais durante as 24 horas. Outra intervenção que devia ser realizada na unidade era a educação dos pais sobre o seu papel relativamente à participação no cuidado de seu filho ${ }^{(49)}$.

Outro estudo constatou equilíbrio entre o teorizado e a qualidade de assistência dos enfermeiros como principal achado para a realização do cuidado centrado na família. Esse equilíbrio foi caracterizado principalmente em situações como: horário de visitas e presença da família no leito e as transformações físicas na unidade de um espaço aberto compartilhado para os quartos individuais privados. Esse estudo ainda enfatizou a importância de os enfermeiros estarem envolvidos nas decisões sobre a melhor forma de implementar o cuidado centrado na família ${ }^{(50)}$.

Por outro lado, outro estudo identificou a existência, em outra UTIP, de uma grande divergência entre as práticas defendidas nos cuidados centrados na família, como teorizado e descrito na literatura pediátrica, e as experiências vividas pelos membros da família ${ }^{(51)}$.

Um fator limitador deste estudo foi que, por meio da busca aleatória, muitos artigos relevantes para a temática, escritos em sua maioria por arquitetos especialistas em edificações de saúde, foram encontrados. Entretanto, pelo fato de terem sido publicados em Anais de eventos científicos, não aparecem nas fontes de informação utilizadas na busca.

\section{Conclusão}

Conclui-se que as UTIP ainda se apresentam, na maioria dos casos, como unidades voltadas ao tratamento curativo das patologias. Nesses locais, os profissionais trabalham, de forma exaustiva e sob condições favoráveis ao estresse, dificultando a implementação do cuidado centrado na criança e em sua família, principalmente no estabelecimento de uma relação harmônica entre família e equipe.

Evidenciam-se também vantagens, principalmente relacionadas ao sono das crianças internadas e ao relacionamento entre equipe e família, nas unidades com quartos individuais em relação às de quartos compartilhados.

Além disso, no que se refere ao ambiente físico da UTIP, constata-se que os profissionais ainda se preocupam pouco com a influência dos elementos ambientais no processo de recuperação da criança, uma vez que o único elemento abordado nos estudos foi o ruído. Por isso, são necessários mais estudos relacionados aos outros elementos, que contemplem conforto e segurança durante a internação na UTIP, tais como iluminação, ventilação, temperatura, espaço físico, utilização de artes, lazer e caracterizações infantis na unidade, permitindo uma assistência mais humanizada para a criança e sua família.

Esta revisão mostrou a necessidade de novas investigações sobre a temática da ambiência da UTIP, para permitir a construção de um arcabouço teórico-prático imprescindível para a implementação da assistência centrada na criança e em sua família nesse espaço de atendimento tão peculiar e estigmatizado, que é a terapia intensiva.

Diante do exposto, os resultados deste estudo contribuirão para nortear as atividades desenvolvidas na UTIP, alicerçar a qualificação e direcionar a prática assistencial, além de organizar os espaços de atendimento à criança e à sua família. 


\section{Colaborações:}

1 - concepção, projeto, análise e interpretação dos dados: Soraya Bactuli Cardoso, Isabel Cristina dos Santos Oliveira, Elena Araújo Martinez, Sandra Alves do Carmo, Rita de Cássia Melão de Moraes e Mauro César de Oliveira Santos;

2 - redação do artigo e revisão crítica relevante do conteúdo intelectual: Soraya Bactuli Cardoso e Isabel Cristina dos Santos Oliveira;

3 - aprovação final da versão a ser publicada: Soraya Bactuli Cardoso.

\section{Referências}

1. Ouchi JD, Lupo APR, Alves BO, Andrade RV, Fogaça MB. O papel do enfermeiro na unidade de terapia intensiva diante de novas tecnologias em saúde. Rev Saúde Foco [Internet]. 2018 [cited 2019 Oct 21];10:412-28. Available from: http://portal.unisepe.com.br/unifia/wp-content/ uploads/sites/10001/2018/07/054_O_PAPEL_DO_ ENFERMEIRO_NA_UNIDADE_DE_TERAPIA_ INTENSIVA.pdf

2. Hospital Infantil e Pronto Socorro Sabará. Primeira UTI pediátrica do Brasil [Internet]. São Paulo; 2018 [cited 2018 Sep 14]. Available from: http://www. hospitalinfantilsabara.org.br/pesquisa-e-inovacao/ primeira-uti-pediatrica-do-brasil/

3. Ramos DZ, Lima CA, Leal ALR, Prado PF, Oliveira VV, Souza AAM, et al. A participação da família no cuidado às crianças internadas em unidade de terapia intensiva. Rev bras Prom Saúde. 2016;29(2):189-96. DOI: http://dx.doi. org/10.5020/18061230.2016.p.189

4. Azevedo AVS, Lançoni Júnior AC, Crepaldi MA. Interação equipe de enfermagem, família, e criança hospitalizada: revisão integrativa. Ciênc saúde coletiva. 2017 nov;22(11):3653-66. DOI: 10.1590/1413-812320172211.26362015

5. Nightingale F. Notas sobre enfermagem: o que é e o que não é. São Paulo: Cortez; 1989.

6. Brasil. Lei n. 13.257, de 8 de março de 2016. Dispõe sobre as políticas públicas para a primeira infância e altera a Lei n. 8.069, de 13 de julho de 1990 (Estatuto da Criança e do Adolescente), o Decreto-Lei n. 3.689, de 3 de outubro de 1941 (Código de Processo Penal), a Consolidação das
Leis do Trabalho (CLT), aprovada pelo Decreto-Lei n. 5.452 , de $1^{\circ}$ de maio de 1943 , a Lei n. 11.770, de 9 de setembro de 2008, e a Lei n. 12.662, de 5 de junho de 2012 [Internet]. Brasília (DF); 2016 [cited 2019 Oct 23]. Available from: http://www. planalto.gov.br/ccivil_03/_ato2015-2018/2016/lei/ 113257.htm

7. Brasil. Ministério da Saúde. A experiência da diretriz de Ambiência da Política Nacional de Humanização (PNH) [Internet]. Brasília (DF); 2017 [cited 2018 Sep 1]. Available from: http://redehumanizasus. net/wpcontent/uploads/2017/09/experiencia_ diretriz_ambiencia_humanizacao_pnh.pdf

8. Olea PM, Ganzer PP, Nodari CH, Lago JD, Dorion ECH, Reis ZC, et al. Análise crítica dos ambientes hospitalares: um estudo de caso em um Hospital da Região da Serra Gaúcha. In: Congresso Brasileiro de Administração, da Saúde e Agronomia. Gestão, Educação e Promoção da Saúde, 2, 2013, São Paulo. Anais (on-line) São Paulo: Convibra; 2013. [cited 2018 Oct 14]. Available from: http://www. convibra.com.br/publicacoes.asp

9. Bergan C, Bursztyn I, Santos MCO, Tura LFR. Humanização: representações sociais do hospital pediátrico. Rev Gaucha Enferm. 2009;30(4):656-61. DOI: http://dx.doi.org/10.1590/ S1983-14472009000400011

10. Sousa LMM, Marques-Vieira CMA, Severino SSP, Antunes AV. A metodologia de revisão integrativa da literatura em enfermagem. Rev Invest Enferm [Internet]. 2017 [cited 2019 Oct 22];2:17-26. Available from: https://www.researchgate.net/ publication/321319742_Metodologia_de_Revisao_ Integrativa_da_Literatura_em_Enfermagem

11. CEBM. Oxford Centre for Evidence-based Medicine - Levels of Evidence (March 2009) [Internet]. Reino Unido; 2009 [cited 2018 Nov 28]. Available from: http://www.cebm.net/oxford-centre-evidencebased-medicine-levels-evidence-march-2009

12. Ribeiro JP, Gomes GC, Thofehen MB. Ambiência como estratégia de humanização da assistência na unidade de pediatria: revisão sistemática. Rev Esc Enferm USP. 2014;48(3):530-9. DOI: http://dx.doi. org/10.1590/S0080-623420140000300020

13. Kudchadkar SR, Beers MC, Ascenzi JA, Jastaniah E, Punjabi NM. Nurses' perceptions of pediatric intensive care unit enviroment and work experience after transition to single-patient rooms. Am J Crit Care. 2016;25(5):e98-e107. DOI: http:// dx.doi.org/ 10.4037/ajcc2016463 
14. Associação Brasileira de Normas Técnicas. NBR 10152 - Níveis de ruído para o conforto acústico. Rio de Janeiro; 1987.

15. Associação Brasileira de Normas Técnicas. NBR 10151 - Avaliação do ruído em áreas habitadas, visando o conforto da comunidade. Rio de Janeiro; 2000 .

16. World Health Organization. Guidelines for community noise. Guidelines values. Geneva; 1999.

17. Carvalho WB, Pedreira MLG, Aguiar MAL. Nível de ruídos em uma unidade de cuidados intensivos pediátricos. J Pediatr. 2005;81(6):495-8. DOI: http:// dx.doi.org/10.1590/S0021-75572005000800015

18. Oliveira FMCN, Paiva MB, Nascimento MAL, Rezende, VM, Silva AS, Silva CRL. Nível de ruído da UTIP: estudo observacional. $\square$ Online Braz J Nurs. 2013;12(3):431-41. DOI: http://dx.doi. org/10.5935/1676-4285.20134043

19. Shoemark H, Harcourt E, Arnup SJ, Hunt RW. Characterising the ambient sound enviroment for infants in intensive care wards. J Paediatr Child Health. 2016;52(4):436-40. DOI: http://dx.doi.org/ 10.1111/jpc. 13084

20. Hickman RL. Evidence-Based Review and Discussion points. Am J Crit Care. 2015;24(5):512-3. DOI: http://dx.doi.org/10.4037/ajcc2015802

21. Bailey E, Timmons S. Noise levels in PICU: an evaluative study. Pediatr Nurs. 2005;17(10):22-6. DOI: $10.7748 /$ paed.17.10.22.s21

22. Watson J, Kindtler A, Vidonish WP, Wagner $\mathrm{M}$, Lin $\mathrm{L}$, Davis KG, et al. Impact of noise on nurses in pediatric intensive care units. Am J Crit Care. 2015;24(5):377-84. DOI: http://dx.doi.org/ $10.4037 /$ ajcc 2015260

23. Kaur H, Rohlik GM, Nemergut ME, Tripathi S. Comparison of staff and family perceptions of causes of noise pollution in the pediatric intensive care unit and suggested intervention strategies. Noise Health. 2016;18(81):78-84. DOI: http:// dx.doi.org/10.4103/1463-1741.178480

24. Alves DFS, Guirardello EB. Ambiente de trabalho da enfermagem, segurança do paciente e qualidade do cuidado em hospital pediátrico. Rev Gaucha Enferm. 2016;37(2):e58817. DOI: http:// dx.doi.org/10.1590/1983-1447.2016.02.58817

25. Alves DFS. Ambiente de trabalho da enfermagem e segurança do paciente em unidades pediátricas [tese]. Campinas (SP): Universidade Estadual de Campinas; 2015.

26. Lake ET, Cordova PB, Barton S, Singh S, Agosto PD, Ely B, et al. Missed Nursing care in Pediatrics. Hosp Pediatr. 2017 Jul;7(7):378-84.

27. Fogaça MC, Carvalho WB, Nogueira PCK, Martins LAN. Estresse ocupacional e suas repercussões na qualidade de vida de médicos e enfermeiros intensivistas pediátricos e neonatais. Rev bras ter intensiva. 2009 Aug;21(3):299-305. DOI: http:// dx.doi.org/10.1590/S0103-507X2009000300010

28. Peixoto TC. UTIP: vidas pulsando entre a estabilidade orgânica e a instabilidade subjetiva [tese]. Belo Horizonte: Universidade Federal de Minas Gerais; 2016.

29. Rosenberg DI, Moss MM, American College of Critical Care Medicine of the Society of Critical Care Medicine. Guidelines and levels of care for pediatric intensive care units. Crit Care Med [Internet]. 2004 Oct [cited 2018 Oct 28];32(10):211727. Available from: https://www.ncbi.nlm.nih.gov/ pubmed/15483423

30. Slusher TM, Kiragu AW, Day LT, Bjorklund AR, Shirk A, Johannsen C, et al. Pediatric Critical care in Resource-Limited Settings-Overview and Lessons Learned. Front Pediatr. 2018 Mar 16;6:49. DOI: $10.3389 /$ fped.2018.00049

31. Calvett PU, Silva LM, Gauer GJC. Psicologia da saúde e criança hospitalizada. Psic [Internet]. 2008 dez [cited 2018 Sep 28];9(2):229-34. Available from: http://pepsic.bvsalud.org/scielo.php?script=sci_ arttext\&pid=\$1676-73142008000200011

32. Baker C. Preventing ICU syndrome in children. Paediatr Nurs. 2004 Dec;16(10):32-5. DOI: 10.7748/ paed2004.12.16.10.32.c885

33. Gomes GC, Erdman AL, Busanello J. Refletindo sobre a inserção da família no cuidado à criança hospitalizada. Rev enferm UERJ [Internet]. 2010 [cited 2018 Sep 20];18(1):143-7. Available from: http://www.facenf.uerj.br/v18n1/v18n1a25.pdf

34. Soares LG, Rosa NM, Molina RCM, Higarashi IH, Marcon SS. UTI pediátrica: o significado do cuidar na perspectiva da mãe. J res: fundam care. online. 2016 out/dez;8(4):4965-71. DOI: 10.9789/21755361 . 2016.v8i4.4965-4971

35. Molina RCM, Marcon SS. Sofrimento psíquico interferindo no desejo materno de cuidar do filho internado na UTIP. Online braz J nurs [Internet]. 2009 [cited 2018 Oct 13];8(3). Available from: 
http://www.objnursing.uff.br/index.php/nursing/ article/view/j.1676-4285.2009.2590/568

36. Reis SMG, Leite ACA, Alvarenga WA, Araújo JS, Zago MMF, Nascimento LC. Metassíntese sobre o homem como pai e cuidador de um filho hospitalizado. Rev Latino-Am Enferm. 2017;25:e2922. DOI: 10.1590/1518-8345.1850.2922

37. Soares JDD, Brito RS, Carvalho JBL. A presença do pai/acompanhante no âmbito hospitalar: revisão integrativa. Rev Enferm UFPE on line. 2014;8(7):2095-106. DOI: 10.5205/reuol.596351246-1-RV.0807201435

38. Smith AB, Hefley GC, Anand KJ. Parent bed spaces in the PICU: effect on parental stress. Pediatr Nurs. 2007 May-Jun;33(3):215-21.

39. Stremler R, Dhukai Z, Wong L, Parshuram C. Factors influencing sleep for parents of critically ill hospitalised children: a qualitative analysis. Intensive Crit Care Nurs. 2011 Feb;27(1):37-45. DOI: 10.1016/j.iccn.2010.11.001

40. Baird J, Davies B, Hinds PS, Baggott C, Rehm RS. What impact do hospital and unit-based rules have upon patient and family-centered care in the pediatric intensive care unit? J Pediatr Nurs. 2015 Jan-Feb;30(1):133-42. DOI: 10.1016/ j.pedn.2014.10.001

41. Lima FET, Jorge MSB, Moreira TMM. Humanização hospitalar: satisfação dos profissionais de um hospital pediátrico. Rev bras enferm. 2006 May-Jun;59(3):291-6. DOI: http://dx.doi.org/ 10.1590/S0034-71672006000300008

42. Reis LS, Silva EF, Waterkemper R, Lorenzini E, Cecchtto FH. Percepção da equipe de enfermagem sobre humanização em Unidade de Tratamento Intensivo Neonatal e Pediátrica. Rev Gaucha Enferm. 2013;34(2):118-24. DOI: http://dx.doi. org/10.1590/S1983-14472013000200015

43. Amaral LFP, Calegari T. Humanização da assistência de enfermagem à família na UTIP. Cogitare Enferm. 2016 jul/set;21(3):1-9. DOI: http://dx.doi. org/10.5380/ce.v21i3.4

44. Abuqamar M, Arabiat DH, Holmes S. Parents' Perceived Satisfaction of Care, Communication and Environment of the Pediatric Intensive Care Units at a Tertiary Children's Hospital. J Pediatr
Nurs. 2016 May-Jun;31(3):e177-84. DOI: 10.1016/j. pedn.2015.12.009

45. Stouffer JW, Shirk BJ, Polomano RC. Practice guidelines for music interventions with hospitalized pediatric patients. J Pediatr Nurs. 2007 Dec;22(6):448-56. DOI: 10.1016/j.pedn.2007.04.011

46. Majdalani MN, Doumit MA, Rahi AC. The lived experience of parents of children admitted to the pediatric intensive care unit in Lebanon. Int $\mathrm{J}$ Nurs Stud. 2014 Feb;51(2):217-25. DOI: 10.1016/j. ijnurstu.2013.06.001

47. Ramírez M, Navarro S, Clavería C, Molina Y, Cox A. Estresores Parentales em Unidad de Cuidados Intensivos Pediátricos. Rev Chil Pediatr. 2018 abr;89(2):182-9. DOI: http://dx.doi. org/10.4067/s0370-41062018000100185

48. Carnevale FA, Gaudreault J. The experience of critically ill children: A phenomenological study of discomfort and comfort. Dynamics [Internet]. 2013 Spring [cited 2018 Oct 23];24(1):19-27. Available from: https://www.ncbi.nlm.nih.gov/ pubmed/23691718

49. Vasli P, Dehghan-Nayeri N, Borim-Nezhad L, Vedadhir A. Dominance of paternalism in family-centered care in the pediatric intensive care unit (PICU): an ethnographic study. Issues Compr Pediatr Nurs. 2015 Jun;38(2):118-35. DOI: 10.3109/01460862.2015.1035464

50. Coats H, Bourget E, Starks H, Lindhorst T, SaikiCraighill S, Curtis JR, et al. Nurses' Reflections on Benefits and Challenges of Implementing FamilyCentered Care in Pediatric Intensive Care Units. Am J Crit Care. 2018 Jan;27(1):52-8. DOI: 10.4037/ ajcc 2018353

51. Macdonald ME, Liben S, Carnevale FA, Cohen SR. An office or a bedroom? Challenges for familycentered care in the pediatric intensive care unit. J Child Health Care. 2012 Sep;16(3):237-49. DOI: $10.1177 / 1367493511430678$

Recebido: 13 de setembro de 2019

Aprovado: 12 de novembro de 2019

Publicado: 4 de fevereiro de 2020 
Soraya Bactuli Cardoso, Isabel Cristina dos Santos Oliveira, Elena Araújo Martinez, Sandra Alves do Carmo, Rita de Cássia Melão de Moraes, Mauro César de Oliveira Santos

A Revista Baiana de Enfermagem utiliza a Licença Creative Commons - Atribuição-NãoComercial 4.0 Internacional. https://creativecommons.org/licenses/by-nc/4.0/

Este artigo é de acesso aberto distribuído sob os termos da Licença Creative Commons (CC BY-NC).

Esta licença permite que outros remixem, adaptem e criem a partir do seu trabalho para fins não comerciais. Embora os novos trabalhos tenham de lhe atribuir o devido crédito e não possam ser usados para fins comerciais, os usuários não têm de licenciar esses trabalhos derivados sob os mesmos termos. 